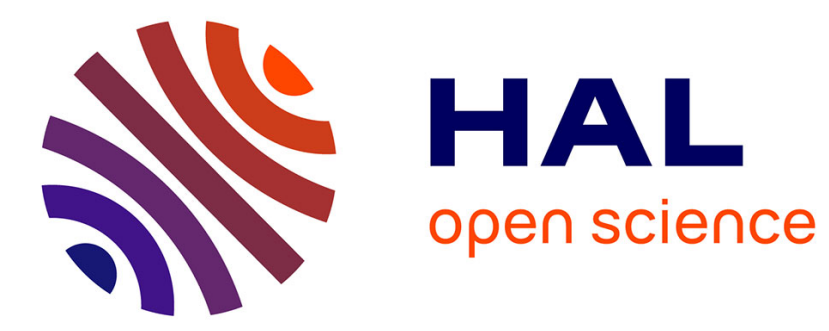

\title{
Les institutions créatrices de proximités
}

Damien Talbot

\section{To cite this version:}

Damien Talbot. Les institutions créatrices de proximités. Revue d'économie régionale et urbaine, 2008, octobre (3), pp.289. 10.3917/reru.083.0289 . hal-02364234

\section{HAL Id: hal-02364234 \\ https://hal.science/hal-02364234}

Submitted on 14 Nov 2019

HAL is a multi-disciplinary open access archive for the deposit and dissemination of scientific research documents, whether they are published or not. The documents may come from teaching and research institutions in France or abroad, or from public or private research centers.
L'archive ouverte pluridisciplinaire HAL, est destinée au dépôt et à la diffusion de documents scientifiques de niveau recherche, publiés ou non, émanant des établissements d'enseignement et de recherche français ou étrangers, des laboratoires publics ou privés. 


\title{
LES INSTITUTIONS CREATRICES DE PROXIMITES
}

\author{
Institutions as creators of proximities
}

\author{
Revue d'Economie Régionale et Urbaine \\ 2008, n’3, pp. 289-310.
}

\author{
Damien Talbot \\ Maître de conférences \\ GREThA \\ (UMR CNRS 5113) \\ Université Montesquieu Bordeaux IV \\ Avenue Léon Duguit 33608 PESSAC CEDEX \\ Tél. : $0556848671 \quad$ Fax. : 0556848647 \\ damien.talbot@u-bordeaux4.fr
}

\section{Résumé}

En mobilisant les apports des institutionnalistes américains et des philosophes de l'esprit, nous proposons un approfondissement des définitions de deux des trois formes canoniques de la proximité. Après avoir rappelé que la proximité géographique est une disponibilité relationnelle, la proximité institutionnelle est définie comme un effet des institutions : elle renvoie à des significations communes à partager et à la fixation de rôles complémentaires à jouer dans l'action collective. La proximité organisationnelle, suivant la distinction proposée par Commons entre institution et organisation, est une forme particulière de proximité institutionnelle qui articule coordination cognitive et coordination politique. Elle consiste pour des acteurs à intégrer la communauté cognitive, c'est-à-dire à accéder à la mémoire de l'organisation composée de règles et de routines, et à intégrer la communauté politique, c'està-dire à s'insérer dans la structure de pouvoir.

Mots clés : proximités, institutionnalisme américain, philosophie de l'esprit.

\section{Summary}

Using contributions of American institutionalists and philosophers of mind, we propose to look further into the definitions of the three traditional forms of the proximity. First, we define geographical proximity as relational availability. Second, we explain that institutional proximity is an effect of institutions. In this case, actors share common significances and play complementary roles in collective action. Third, organisational proximity, according to distinction suggested by Commons between institution and organization, is understood as a particular form of institutional proximity which articulates cognitive coordination and political coordination. Actors integrate cognitive community, i.e. reach memory of organization made up of rules and routines, and integrate political community, i.e. take place in power structure.

Key words: proximities, american institutionalism, philosophy of mind.

Classification JEL: B52, R39. 


\section{Introduction}

Le terme "proximité" connaît, au moins en France, un vif succès dans divers domaines : on parle à la fois de police de proximité, de justice de proximité, de démocratie de proximité, de services de proximité, etc., comme si des relations sociales par trop distendues devaient être rapprochées. Implicitement, l'hypothèse est faite que la proximité présenterait des caractéristiques favorisant le maintien, voire le renforcement, des relations, quel que soit le domaine d'activité. Les relations économiques (marchandes et non-marchandes) n'échapperaient pas à cet état de fait. L'engouement international actuel que connaît la notion de cluster, à la fois chez les décideurs politiques et les chercheurs en économie, trouve son fondement dans cette hypothèse implicite.

De quelle proximité parle-t-on ? Intuitivement, l'on pense en premier lieu à la proximité géographique qui réunit des agents partageant un même espace. Si la théorie économique a longtemps considéré l'espace comme une variable neutre, il est aujourd'hui établi qu'elle devait être prise en compte dans l'analyse. Partant du constat que lorsque les agents sont proches géographiquement leurs relations économiques peuvent s'en trouver affectées, les chercheurs groupe "Dynamiques de Proximité"1 ont plaidé avec d'autres, dés le début des années 90 , pour une telle endogénéisation de l'espace et pour une intégration dans la théorie économique.

Il apparaît en second lieu que l'agent est présent à la fois « ici et ailleurs » (RALLET, 1999). Ici car il est localisé dans un espace géographique au sein duquel il entretient des relations de voisinage, ailleurs car l'acteur est évidemment en relation à distance avec d'autres agents. De fait, on peut être « proche » de quelqu'un tout en étant éloigné géographiquement : la proximité présente alors, au côté de la dimension spatiale, une dimension non spatiale de type relationnel.

Il faut ensuite préciser le contenu de ces proximités. Plus que de «proximité », il faut ici parler de « coordination par la proximité ». Vouloir appréhender la proximité permet de poser des questions du type « qui est proche de qui ? », « qui est proche de quoi ? », ou encore " que faut-il partager pour agir ensemble ? ». Cet article cherche à répondre, au moins en partie, à cette dernière question. Alors la proximité renvoie au fait pour des acteurs de partager des dispositifs de coordination. Plus précisément, il est possible de distinguer deux dispositifs essentiels de coordination, en échos aux dimensions spatiale et non spatiale de la proximité : l'espace géographique d'une part, les institutions (englobant dans leur définition les organisations, $c f$. infra) d'autre part.

La première partie de cette affirmation fait l'objet d'un large consensus au sein du groupe «Dynamiques de Proximité »; il n'en est pas de même de la seconde partie. Un premier courant qualifié d'interactionniste se limite à deux formes de proximité (géographique, organisée), tandis qu'un second d'inspiration institutionnaliste décline la proximité en trois composantes (géographique, institutionnelle, organisationnelle). L'approche interactionniste ne traite du partage des institutions que de façon implicite, tandis que l'approche institutionnaliste accorde à ce partage une place essentielle, qui nous conduit à en faire une catégorie de la proximité à part entière. Nous prenons part à ce débat en expliquant pourquoi il est nécessaire de conserver la distinction entre proximité institutionnelle et proximité organisationnelle. L'argument essentiel que nous aurons à développer souligne qu'une

\footnotetext{
${ }^{1}$ De façon non exhaustive, le lecteur peut se référer à RERU (1993), GILLY et TORRE (2000), PECQUEUR et ZIMMERMANN (2004), TORRE et FILIPPI (2005), TALBOT et KIRAT (2005) ou encore TORRE et RALLET (2005).
} 
conception interactionniste de la proximité conduit à ne pas traiter la dimension politique de la coordination, qui se veut complémentaire de la dimension cognitive. C'est en quelque sorte le «prix à payer » lorsqu'on n'aborde pas frontalement la question du partage des institutions comme condition à l'action collective. Or, l'action collective suppose pour se réaliser le partage, certes de croyances et d'une vision commune du monde, mais aussi d'accords, de compromis transitoires face à la divergence des intérêts. Le traitement simultanée de ces espaces de sens (dimension cognitive) et de choix (dimension politique) en économie est notamment le fait des approches institutionnalistes, tout particulièrement des institutionnalistes américains, grâce à l'introduction du concept d'institution. En effet, et nous aurons à le montrer, l'institution n'est pas un simple dispositif cognitif de coordination ; elle permet aussi de régler les conflits issus de l'hétérogénéité des agents en termes de goûts, de richesses, de compétences, d'anticipations, etc., ce qui lui donne une nature fondamentalement politique (AMABLE, 2005).

Nous entamons ce travail en définissant la proximité géographique, conçue comme une disponibilité relationnelle (section 1). Nous poursuivons en expliquant que l'interactionnisme est une approche qui ne facilite pas l'intégration des conditions d'ordre politique à l'action collective, nous conduisant à l'adoption d'une perspective institutionnaliste (section 2). Il nous faut ensuite définir précisément la proximité institutionnelle, ce qui suppose en préalable un effort de définition du concept d'institution (section 3), pour ensuite affirmer que les institutions sont génératrices d'une proximité, dite institutionnelle (section 4). Sur cette base, nous définissons, dans une perspective commonsonienne, la proximité organisationnelle comme une forme particulière de proximité institutionnelle, puisque l'organisation est un phénomène d'abord institutionnel (section 5).

\section{- 1 - \\ La proximité géographique : une disponibilité relationnelle}

Nous partons de la forme la plus intuitive de la proximité, dite géographique, qui aborde la question des conditions objectives de localisation (PECQUEUR et ZIMMERMANN, 2004). A la croisée de l'Economie Régionale et de l'Economie Industrielle, l'approche par la proximité traite de façon originale les questions de localisation des firmes et des activités, en particulier au regard des analyses spatiales qui abordent la question des choix de localisation comme l'unique résultat d'un calcul optimisateur, ou encore de l'Economic Geography (KRUGMAN, 1991) pour laquelle le problème de la localisation des unités de production est expliqué par l'existence de rendements croissants. Les analyses en termes de proximités, en posant la question du rôle de l'espace géographique dans les coordinations entre acteurs, assimilent le partage d'un même espace géographique à une condition permissive pour la coordination. En cela, elles s'inscrivent dans la tradition marshallienne des districts industriels, au côté des théories sur les systèmes nationaux d'innovation (LUNDVALL, 1992), sur les systèmes productifs locaux (COURLET et SOULAGE, 1994), sur les milieux innovateurs (CAMAGNI et MAILLAT, 2006), ou encore sur les clusters (PORTER, 1998), pour qui la proximité géographique entre firmes est perçue comme un facteur de diffusion des informations et des connaissances. Elles s'en démarquent toutefois en insistant sur le fait que les conditions à la coordination, comme par exemple l'existence d'une confiance entre les acteurs, ou bien les formes d'action collectives qui en résultent à l'instar des territoires, sont des construits. L'approche par la proximité cherche à rendre compte de ce processus de construction par l'analyse des interactions, qui deviennent l'unité d'analyse de base. C'est alors la place de la proximité géographique dans ce processus de construction qui est questionnée. 
La proximité géographique est une représentation portée par des individus de la distance qui séparent dans l'espace des objets et/ou des individus. Comme jugement porté sur une distance, elle est doublement relative (TORRE et RALLET, 2005 ; TORRE et FILIPPI, 2005) en raison des caractéristiques propres à l'espace géographique :

- relative car la distance est pondérée par le temps et les coûts de transports, du fait de la structuration matérielle de l'espace, ce qui renvoie à l'idée classique selon laquelle l'espace physique est structuré par des infrastructures de transport et de communication, constituant un cadre matériel favorisant la circulation des informations, des biens physiques et des individus ;

- relative car la distance qui sépare des individus, des organisations ou des villes est aussi une représentation, un jugement conduisant à se situer de façon binaire « près de » ou « loin de ». Parce que les acteurs co-localisés partagent un certain sens commun du fait d'une localisation identique, les individus qui habitent un même lieu ont de facto une même référence. L'espace est ainsi porteur d'une signification intersubjective, au sens de DESCOMBES ${ }^{2}$ (1996). Les significations intersubjectives correspondent aux consensus entre des individus indépendants. C'est par exemple le cas des similitudes entre divers jugements de goûts. Cette similitude des jugements peut s'expliquer par les conditions historiques (éducation, action publicitaire, recherche d'un consensus, etc.). Il n'en reste pas moins qu'il s'agit d'une libre rencontre de jugements indépendants, reposant sur «un universel de ressemblance » selon les termes du philosophe (ibid., p. 292). Dans notre cas, la similitude de jugement porte sur le nom de cet espace, ses limites physiques, son histoire, son patrimoine, les usages et les modes de vie qui y prennent place, les coordinations précédentes réussies ou échouées, etc. L'espace géographique, porteur d'une telle signification, devient alors un lieu particulier. Il constitue un référent cognitif et il intervient dans le processus de construction (ou de destruction) des identités dans le sens où il est une composante du rapport aux autres (PECQUEUR, 1997), puisque les acteurs locaux le font exister au regard des autres (LAGANIER, VILLALBA et ZUINDEAU, 2004).

Le fait de se sentir «proche» ne doit pas être interpréter comme l'assurance qu'une relation s'instaure. Les significations intersubjectives faisant par définition l'objet d'un consensus sont aisément communicables, mais la relation à autrui ainsi posée n'est ici qu'une possibilité de relation entre deux individus pour l'instant indépendants. Cette ressemblance ne repose sur aucune connexion positive des individus et n'implique pas une relation systématique. La même remarque peut être faite au sujet de la structuration matérielle de l'espace : une route n'implique pas une interaction. Cela signifie simplement que l'existence d'une forte proximité géographique entre acteurs n'est pas neutre : elle est une mise en disponibilité relationnelle, une ressource encore latente ${ }^{3}$.

A ce stade, aucune connexion réelle localisée n'est encore établit. Celle-ci suppose d'autres formes de proximités non spatiales pour prendre corps. Deux approches sont à distinguer,

\footnotetext{
${ }^{2}$ Nous ferons appel tout au long de ce travail aux apports intentionnalistes de l'esprit, comme Descombes, qui, comme les institutionnalistes américains, fonde sa compréhension de l'institution sur la philosophie pragmatique développée par Pierce.

${ }^{3}$ Cette possibilité de relation ne préjuge en rien de son contenu, dans le cas où elle se noue. La littérature sur les conflits d'usage de l'espace ( $c f$. notamment CARON et TORRE, 2005 ; JEANNEAUX et KIRAT, 2005) cite à ce propos nombre d'exemples d'inégalités liées à la situation topographique des acteurs (un usager subit la pollution de l'eau provenant d'un autre usager situé en amont). Elle démontre que la proximité géographique, quand elle est subit, peut être constitutive d'inégalités créant des rapports de force entre les acteurs.
} 
entre les tenants d'une vision interactionniste de la proximité, qualifiée alors d'organisée, et les tenants d'une vision institutionnaliste de la proximité, qui distinguent la proximité institutionnelle de la proximité organisationnelle. La première présente à nos yeux une limite essentielle exposée dans la section suivante.

\section{La proximité organisée : la dimension politique occultée}

Nous abordons ici la question de la définition du versant relationnel de la proximité qui, comme la proximité géographique, doit être comprise à la fois comme un potentiel qui peut faciliter des coordinations et une contrainte (limites culturelles à la coopération, externalités négatives, etc.) (PECQUEUR et ZIMMERMANN, 2004). Une définition dite « interactionniste » est généralement proposée.

\subsection{Une définition interactionniste de la proximité}

La proximité organisée se définit par la capacité qu'offre une organisation de faire interagir ses membres (TORRE et RALLET, 2005). Cette capacité est le résultat à la fois d'une logique d'appartenance qui traduit le fait que les membres d'une organisation interagissent effectivement grâce à l'existence de règles et de routines de comportements et d'une logique de similitude qui exprime la communauté de croyances et de savoirs que lie les participants. Cette proximité organisée peut révéler les effets positifs, mais aussi négatifs, de la proximité géographique (TORRE et FILIPPI, 2005) et elle exprime le passage d'une co-localisation à une coordination, à l'instar du cas particulier du territoire dans lequel se déploie une action collective localisée.

Cette définition de la proximité organisée suppose l'existence d'interactions entre acteurs, entre objets techniques ou entre acteurs et objets. Elle s'inscrit dans une perspective interactionniste qui, au côté des approches individualistes et holistes, constitue une troisième voie. L'interactionnisme conçoit la société comme une composition des relations interindividuelles et prend pour unité de base de l'analyse l'interaction, conçue comme un « champ mutuel d'influence » (LE BRETON, 2004, p. 51), des actions qui se déterminent les unes les autres. Sans voir ses comportements strictement déterminés par des éléments extérieurs, l'individu n'est pas non plus une monade détachée de toute influence: "indéterminée dans son mouvement, [l'interaction] s'établit néanmoins sur un canevas d'attentes mutuelles » (ibid., p. 52). Il construit son univers de sens, non pas en fonction d'attributs ou d'une imposition extérieure, mais à travers une activité délibérée de donation de sens, une capacité d'interprétation des normes et des règles. L'individu donne un sens à ses actions et à leurs conséquences, fait de même avec celles des autres, et agit. La réciprocité des perspectives est alors une condition au fait d'agir socialement: l'individu, dans son comportement, s'impose de prévoir comment les autres vont réagir. Inversement, lorsque les autres agissent à son propos, il connaît la gamme de comportements que ceux-ci attendent en retour.

Cette approche présente l'avantage par rapport à l'individualisme méthodologique de pouvoir saisir les relations fondées sur le fait que les termes relatifs sont indissociables. Autrement dit, l'interactionnisme met à jour une connexion permettant d'attribuer à chaque partie reliée un caractère qui dépend de la relation. En ce sens, l'interactionnisme ne relève pas de la simple intersubjectivité reliant deux individus possédant une simple communauté de caractère. 
L'autre avantage de l'interactionnisme est de rejeter la figure de l'agent représentatif au profit d'un agent qui tient compte dans son action du comportement des autres agents avec lesquels il entretient des liens directs (GROSSETTI et FILIPPI, 2004). Par conséquent, les interactions délimitent un voisinage au sein des réseaux sociaux, cadre de cette réciprocité, des relations et de la diffusion des informations. Elles deviennent un outil analytique utilisé pour mettre à jour les liens entre modes de coordination, nature des connaissances et processus d'apprentissages, déterminant la co-construction de l'environnement.

Toutefois, face à ces avantages, une critique essentielle peut être proposée, celle de l'oubli de la dimension politique des conditions à la coordination.

\subsection{Réintroduire la dimension politique grâce au concept d'institution}

L'adoption d'un paradigme interprétatif, suggéré par l'interactionnisme, conduit à voir dans la proximité organisée en priorité un lien social de nature principalement cognitive. Cette proximité organisée repose sur une vision partagée du monde, un partage de représentations, de croyances et de savoirs etc., bref une logique de similitude. De même, la logique d'appartenance renvoie au fait que deux membres d'une même organisation sont proches parce qu'ils interagissent dans un même cadre de règles et de routines (TORRE et RALLET, 2005) dont ils partageraient les mêmes interprétations. Il ne s'agit pas de nier l'existence de telles logiques : par contre, nous contestons le fait d'y réduire la proximité non spatiale. Une lecture uniquement cognitive de la proximité ne permet pas de répondre totalement à certaines questions essentielles à la compréhension d'une proximité non-spatiale. Comment se résolvent les conflits inhérents à toute organisation, exemple paradigmatique du déploiement d'une proximité organisée ? Notamment, comment intégrer le rôle des dispositifs juridiques (le Droit) dans cette résolution? Comment se construisent les compromis que suppose l'action collective ? Comment les problèmes sont-ils hiérarchisés ? Comment se construisent les choix collectifs ? Comment s'imposent les décisions collectives ? Comment l'individu s'insère-t-il dans la structure de pouvoir que constitue l'organisation ?

La pensée institutionnaliste cherche à répondre à ces questionnements, considérant que toute coordination suppose en préalable tout autant la construction d'un espace de sens commun que d'un espace de hiérarchisation, de choix et de régulation collective. Adopter une lecture institutionnaliste de la proximité revient à considérer que les conditions à la coordination sont d'ordre cognitif et politique. L'utilisation de l'institution, comme synthèse conceptuelle des conditions cognitives et politiques à la coordination, s'impose alors. L'enjeu n'est pas de faire appel ponctuellement au «contexte institutionnel» comme un «ailleurs sociologique », de réduire l'institution à un signal qui autorise la construction d'anticipations. Nous plaidons pour une endogénéisation des institutions : il s'agit de débuter l'analyse des conditions à la coordination par l'affirmation que le partage d'institutions entre des individus est une condition sine qua non à toute coordination. Cette endogénéisation permet ensuite de mettre à jour le fait que l'institution est créatrice d'une forme de proximité.

Finalement, cette définition de la proximité non spatiale fondée sur une approche interactionniste demeure à nos yeux fondamentalement incomplète pour, en général, rendre compte du rôle des institutions dans les relations de proximité, et en particulier, proposer une définition de la proximité non spatiale qui intègre à la fois une vision cognitive et politique des conditions à la coordination. Pour ce faire, nous proposons l'adoption d'une perspective institutionnaliste en montrant en quoi elle autorise cette double intégration. Mais avant de 
définir les formes non spatiales de la proximité, il est nécessaire de revenir sur le concept d'institution tant ce terme renvoie à des acceptions différentes.

\section{- 3 - \\ Un éclairage sur le concept d'institution}

Nous ne proposons pas ici de réaliser une recension exhaustive des définitions du terme, mais d'expliquer certaines particularités de l'institution mises à jour par les institutionnalistes, notamment américains ${ }^{4}$. Ce courant institutionnaliste ne doit pas être confondu avec celui de la Nouvelle Economie Institutionnelle, dont Williamson, North ou Aoki sont parmi les auteurs emblématiques. Pour ces derniers, les individus dotés d'une rationalité limitée qui évoluent dans un monde incertain doivent faire appel à des institutions pour se coordonner. L'institution joue ici le rôle de réponse théorique à l'introduction de l'incertitude en économie, en guidant les actions individuelles. Elle constitue un substitut ou un complément du marché (SJÖSTRAND, 1995) qui permet la coordination de l'activité économique.

Cette vision de l'institution est développée par WILLIAMSON (1994) qui enseigne qu'en raison de la nature des transactions (incertitude, fréquence des transactions et spécificité des actifs), de la rationalité limitée et du comportement opportuniste des agents, l'échange comporte un coût de transaction. Dès lors, les agents économiques vont créer des structures de gouvernance afin d'économiser ces coûts. Ces structures, qualifiées d'institutions, sont définies par WILLIAMSON (1996), comme "the institutional framework within which the integrity of a transaction, or related set of transactions, is decided" (ibid, p. 397). S'il existe différentes structures de gouvernance, l'institution de référence dans la théorie transactionnelle reste le marché. C'est parce que le marché est imparfait, et donc que son utilisation a un coût, que les agents optent pour d'autres formes de coordination (hiérarchie, réseau), qui apparaissent alors comme une solution au problème de non-optimalité du marché.

North adopte une définition différente de celle proposée par Williamson, même s'il conserve l'idée que les institutions sont des structures qui encadrent les comportements : celles-ci ne sont plus de simples structures organisationnelles, mais se situent à l'aplomb des agents et des organisations. Il existe un premier niveau, celui de la matrice institutionnelle composée d'institutions, et un second niveau, celui des organisations composées d'agents économiques. Par analogie, les institutions sont en quelque sorte les règles d'un jeu, en l'occurrence le jeu économique, dont les joueurs seraient les organisations et les entrepreneurs (NORTH, 1990, 1994).

Plus récemment, note CHAVANCE (2007), North insiste sur le rôle des structures de croyance, rejoignant Aoki dans une analyse d'abord cognitive des institutions. En revanche, ce dernier interprète l'institution, non pas comme une structure, mais comme un équilibre d'un jeu. L'institution est alors un système de croyances partagées sur la manière dont les règles du jeu sont jouées, qui émerge lorsque les agents croient que les autres vont suivre telle ou telle règle d'action les amenant à leur tour à stabiliser leurs propres règles d'actions (AOKI, 2006). Pour chacun, la meilleure réponse possible est de suivre la règle, car l'agent anticipe que les autres agents vont suivre la règle, les autres agents faisant le même calcul que lui. Dans ce cas, l'institution est un équilibre de Nash, les agents n'ayant pas intérêt à dévier de leur stratégie (CHAVANCE, 2007).

Si les institutionnalistes américains voient eux aussi dans les institutions des cadres à l'action collective, ils se démarquent des approches précédentes à la fois par le

\footnotetext{
${ }^{4}$ Parfois aussi nommés « Old Institutionalists ».
} 
positionnement méthodologique choisi et par la définition des institutions retenues. Ainsi, en posant le principe d'antériorité des institutions à toutes actions individuelles, ils adoptent par là-même un positionnement méthodologique plus collectif. L'institution n'est plus uniquement un dispositif de coordination ex post des actions, elle agit en amont de ces dernières en intervenant sur les préférences des agents. En opposition à la démarche où l'institution est une variable expliquée par le marché et les actions individuelles, la causalité est ici inversée : l'institution sera une variable explicative de ces actions et des préférences (RUTHERFORD, 1995). La définition de l'institution proposée par les institutionnalistes américains repose sur deux idées originales : ne pas séparer la cognition de l'action ; l'institution, comme acte collectif, est au service d'une finalité.

\subsection{Une idée commune en acte...}

Il faut constater une relative fluctuation dans la définition de l'institution au sein même du courant institutionnaliste américain. De manière caricaturale, il est possible de distinguer trois groupes d'auteurs selon qu'ils soient affiliés à l'institutionnalisme de Veblen, à celui de Commons où qu'ils adoptent une voie médiane :

- un premier ensemble insiste particulièrement sur la dimension cognitive des institutions : ce sont ici des habitudes de pensée qui constituent des repères de la coordination. Il faut voir dans cette notion d'habitude, non pas un comportement, mais une disposition à penser et à agir de la même façon. VEBLEN $(1898,1899)$ est l'auteur emblématique de cette vision des institutions, qui naissent d'un processus adaptatif et d'une sélection des habitudes mentales devenues par là même prédominantes ;

- sans renier la dimension cognitive des institutions, un deuxième ensemble privilégie l'action dans la définition des institutions, suivant en cela COMMONS (1931), dont l'idée centrale est d'approfondir le mode d'expression organisationnel et politico-juridique des institutions (BAZZOLI et DUTRAIVE, 2002). Commons se distingue de Veblen en plaçant les institutions dans l'ordre des comportements (HODGSON, 2003) et propose une définition centrée sur l'action collective : une institution est une action collective en contrôle et en libération de l'action individuelle ;

- un troisième ensemble d'auteurs contemporains fondent plus explicitement leur définition des institutions sur la double polarité cognition / action. Ainsi, pour BUSH (1987), les institutions sont des structures de comportements reliés par des valeurs. Chez HAMILTON (1993), le concept d'institution renvoie à une façon de penser ou d'agir, encastrée dans les habitudes d'un groupe ou les coutumes d'un peuple. Elles fixent le cadre et la forme des activités humaines, qu'elles soient informelles ou formelles. Les usages informels (coutumes, code moral, etc.) sont des institutions qui sanctionnent et imposent des tabous. De même, toutes les organisations formelles (le gouvernement, l'université, l'Eglise, etc.) exercent une autorité coercitive sur leurs membres. Tous constituent un standard de la conformité : l'individu qui s'y soustrait le fait à ses risques et périls. Cette vision est proche de celle d'HODGSON (2006) qui définit les institutions comme «systems of established and prevalent social rules that structure social interactions. (...) Generally, institutions enable ordered thought, expectation, and action by imposing form and consistency on human activities. They depend upon the thoughts and activities of individuals but are not reductible to them » (p. 2). 
Au-delà de ces divergences, l'unité des institutionnalistes américains se fait sur la nécessité de rejeter le dualisme cartésien entre pensée et action, suivant en cela la philosophie pragmatique développée notamment par Pierce. Le pragmatisme critique le rationalisme cartésien qui suppose "une séparation du monde matériel, régi par des lois, et de la pensée, la raison permettant de découvrir ces lois. Si on sort de ce paradigme, alors les dichotomies deviennent poreuses (...)»(BAZZOLI et KIRAT, 2003, p. 182). Et Bazzoli et Kirat d'ajouter que, dans le pragmatisme de Pierce, les idées ne sont plus des a priori hors du temps, mais des significations produites et re-produites dans un processus d'interaction constant entre la pensée des faits et l'expérience des faits. Cette conception pragmatique réunifie le monde de la pensée et le monde des actes, invalide cette frontière traditionnelle proposée par Descartes entre pensée et action pour en venir à une distinction entre un monde naturel et un monde institutionnel. Il s'agit de connecter le monde mental et le monde extérieur, et de reconnaître avec DESCOMBES (1996) qu'il n'est pas possible de faire abstraction du contexte pour spécifier les pensées.

Dans cette perspective, l'institution est à la fois la règle et le comportement, la représentation et les pratiques, les habitudes de pensée et de faire, sans que les uns ne soient réductibles aux autres. Toute action devient une sorte de langage, traduisant une idée (MAUSS, 1950), la croyance se manifestant dans la conduite. Il s'agit en quelque sorte de « faire » des idées collectives ${ }^{5}$. L'institution devient alors une idée commune en acte.

\section{2. ...au service d'une finalité}

Les institutions sont préétablies, non seulement par des individus à leur propre intention, mais aussi socialement préétablis : en ce sens, elles constituent des attentes normatives définies préalablement à tout exercice et doivent être considérées comme des prêts à pensée et à faire dont les individus ne sont pas a priori les auteurs (DESCOMBES, 1998). Faire appel à une institution, c'est être l'unique auteur de ses actions, mais pas toujours du sens de celles-ci.

Il ne faut pas voir dans cette affirmation un quelconque déterminisme. COMMONS (1934), dans une démarche volitionnelle, fait de l'intention une cause essentielle de l'action humaine. La volonté humaine peut s'exprimer par des décisions particulières ou par le fait de laisser s'instaurer certaines habitudes qui se transforment en coutumes. DESCOMBES $(1996,2004)$ défend lui aussi une conception intentionnaliste de l'esprit. L'intention, notamment collective, instaure une relation entre l'activité présente et un résultat escompté. SEARLE $(1998,2005)$ voit dans l'intentionnalité collective, le présupposé à tout fait social. Cette intentionnalité s'exprime dans l'imposition collective d'une fonction à des entités qui ne peuvent accomplir les fonctions en vertu de leur seule structure physique. Le papier-monnaie vaut de l'argent car nous le souhaitons et nous l'acceptons collectivement. L'acceptation et la reconnaissance d'une fonction est pour cet auteur constitutive d'un fait institutionnel. Il n'existe donc des institutions que par accord humain.

Les institutionnalistes américains franchissent un pas supplémentaire en affirmant que si les institutions sont bien l'œuvre d'une intentionnalité collective, elles apparaissent en outre orientées vers une fin collective en vue de satisfaire les intérêts des acteurs dominants, et doivent d'être examinées en référence à ces mêmes fins. Si les institutions ne peuvent pas avoir de desseins délibérés (DOUGLAS, 2004), elles sont artificielles, profondément humaines et ont un caractère performatif qui lui assure d'ailleurs une certaine pérennité. Commons introduit le concept de futurité (futurity) dans son analyse pour révéler l'importance de cette question des finalités. GISLAIN (2002) explique que, selon Commons,

\footnotetext{
${ }^{5}$ SEARLE $(1998,2005)$ utilise l'expression de «faits mentaux » pour qualifier les faits institutionnels.
} 
les hommes vivent dans le futur et agissent dans le présent. Leurs actions sont fonction de leurs hypothèses habituelles concernant les conséquences futures de ces mêmes actions. La futurité se définit alors comme une représentation perpétuellement actualisée d'un but à atteindre dans le futur par les actions présentes.

\section{-4 - \\ La proximité institutionnelle}

C'est donc une définition de l'institution entendue comme une idée commune en acte au service d'une finalité que nous retenons ici, en vue de donner un contenu à la proximité institutionnelle. Au préalable, il faut rappeler que si toutes les institutions sont sociales par leur source et leur transmission, toutes n'ont pas pour but d'organiser la société (DESCOMBES, 1996). Certaines institutions ont aussi vocation, pour DEFALVARD (1999), à construire les relations de l'individu à la nature brute, défaite de toute métaphysique, morale, théologie ou politique. Au côté des sciences de la nature ainsi définies, les institutions de l'individualité construisent les relations de la vie tournée vers soi. Enfin, certaines institutions, qui attirent notre attention ici, ordonnent la vie sociale. A ce titre, elles sont fondatrices de relations sociales et donc d'une forme de proximité. Pour expliciter ce phénomène de création de proximité, il faut rappeler avec Descombes que les institutions sont révélatrices de l'existence des significations communes aux individus, tout en mettant en relation des acteurs aux rôles complémentaires et aux statuts différents.

\subsection{Significations communes}

Nous avons traité, en première partie de ce travail, la question des significations intersubjectives. Cette conception de la communauté des idées n'épuise pas complètement le sujet de la vie sociale. Prenons avec Descombes un exemple. Comment expliquer l'existence d'actions collectives, comme la négociation ou bien l'élection, qui réunissent des acteurs aux opinions divergentes, non similaires? Dans le cas de l'élection, on vote pour de multiples candidats sans avoir les mêmes opinions politiques que l'ensemble des autres électeurs. Cela signifie que des individus aux significations intersubjectives différentes participent à une même action collective. Le partage des significations intersubjectives ne constitue donc pas une condition impérative à l'action collective. Quelles sont alors ces conditions ?

Il faut alors introduire une autre communauté de pensées, celle des significations communes. Pour que des opinions divergentes puissent s'exprimer, il est indispensable que les participants aient la même représentation de ce qu'ils sont en train de faire, ici voter. Comme l'élection a bien lieu, il faut supposer qu'il existe un sens impersonnel et général de cette pratique, un sens commun défini hors des opinions de chacun et avant que les électeurs aient acquis le sens de cette pratique. Ces significations communes ne sont d'ailleurs pas identiques par coïncidence, mais «sont inculquées aux individus de façon à rendre possible de la part de chacun d'eux des conduites coordonnées et intelligibles du point de vue du groupe » (DESCOMBES, 1996, p. 294). Parce que les acteurs sont cognitivement proches, ils peuvent agir collectivement. Ces significations communes portées par les institutions sont constitutives, avec les significations intersubjectives, d'une proximité dite institutionnelle qui toutefois ne s'y réduit pas. 


\subsection{Des acteurs aux rôles complémentaires}

En effet, les institutions ne mettent pas en présence deux individus sans liens, mais «deux partenaires qui doivent faire des choses différentes et dont les rôles et les statuts sont justement fixés par une règle établie, un usage social que les gens suivent » (DESCOMBES, 1996, p. 297). Ainsi, l'activité sociale de l'enseignant relève d'abord du fait qu'il soit en train d'enseigner. Sans l'action des étudiants qui étudient une matière auprès du professeur, celui-ci peut parler distinctement, il n'enseigne pas. De même, la propriété suppose une relation sociale entre un propriétaire et un locataire ou entre deux propriétaires de terrains mitoyens, bref entre le statut de propriétaire et un autre statut. L'idée de propriété est une représentation collective incarnée dans la relation sociale entre des statuts complémentaires, comme les deux faces d'une même pièce (DESCOMBES, 2000).

Si l'institution qui ordonne la vie sociale relie deux partenaires, il n'est pas toutefois nécessaire qu'il s'agisse d'un rapport entre un particulier et un partenaire identifié comme tel. Ce peut être le rapport entre un particulier et n'importe qui. Le rapport de propriété est principalement un rapport entre quelqu'un (un propriétaire) et quelque chose (l'objet possédé). La relation de particulier à particulier est encore virtuelle et ne sera instauré que si quelqu'un conteste le droit sur l'objet, veut le louer pour son propre usage, etc. A priori, le fait d'être propriétaire ne met pas systématiquement en rapport avec telle ou telle personne : ce n'est qu'une possibilité parmi d'autres. De même, un vendeur peut mettre en vente un bien, sans trouver d'acheteur. Le détenteur de monnaie peut se contenter de thésauriser. En ce sens, l'action collective est en devenir, rendue possible par les significations communes. Il faut voir dans cette remarque une explication au fait que la proximité institutionnelle soit avant tout un potentiel, qui peut être activé par l'effectivité d'une action collective.

\subsection{Une proximité institutionnelle par nature asymétrique}

La complémentarité des rôles des partenaires est intrinsèquement génératrice d'inégalités. De fait, l'utilisation d'institutions peut engendrer des situations d'inégalités et de dépendance à l'origine de conflits. Comme la création de faits institutionnels consiste à imposer un statut et une fonction à une entité (objet, personne, évènement) qui en était dépourvu, SEARLE (2005) considère que cette création revient à imposer un pouvoir, générateur de droits (capacité à) et d'obligations (contraint de). Il s'agit d'imposer des responsabilités, des privilèges, des obligations, des sanctions, des autorisations, etc. Le professeur a le droit d'enseigner, le propriétaire de vendre, le détenteur d'argent d'acheter, le donneur d'ordres de mettre fin à une relation productive, le chef d'entreprise de créer ou de mettre fin à un contrat de travail. Autrement dit, la relation sociale n'est pas a priori équitable et juste. Il s'agit au contraire d'un rapport de force a priori, ce qui explique pourquoi cette relation est par nature conflictuelle. D'autant que les partenaires n'émergent pas ex nihilo afin de se coordonner : il existe une asymétrie de ressources cognitives et matérielles issues de l'histoire des agents, une position sociale, ce qui rend ex ante les termes de l'échange plus favorables à l'une des parties.

Au final, la proximité institutionnelle, comme condition à l'action collective, ne peut renvoyer à la seule existence de significations communes aux acteurs. L'institution génère un autre élément de la proximité institutionnelle, relatif au constat de la fixation par l'institution de rôles complémentaires à jouer dans l'action collective par des acteurs aux positions sociales asymétriques. Mobiliser une institution revient à entrer potentiellement ou effectivement en relation inégale avec autrui, auprès duquel on est dorénavant proche. 
Nous ne réduisons pas la proximité institutionnelle à une seule dimension cognitive renvoyant à une vision partagée du monde ; nous ajoutons à la définition de la proximité institutionnelle une dimension politique régulatrice qui attribue des rôles à des acteurs hétérogènes afin d'apaiser transitoirement les conflits. En ce sens, cette proximité n'est pas du seul ordre de la ressemblance fondé sur une relation entre deux faits et/ou individus distincts et indépendants. Elle est aussi de l'ordre de la communauté qui relie deux faits et/ou individus désormais dépendants, constitués en système. La proximité institutionnelle est irréductible à la seule logique de similitude, pour passer d'une ressemblance de caractère à une connexion positive.

Sur la base de cette proximité institutionnelle, des relations complexes, délimitées et orientées deviennent possibles entre de multiples individus. Il en est ainsi des coutumes, des habitudes collectives, des préjugés, des règles juridiques ou morales mais aussi des parlements, des entreprises, des associations, etc., qui constituent une manifestation concrète des institutions. Ce sont tous des phénomènes fondamentalement de même nature, de l'ordre de l'action sociale qui présente la particularité que si l'autre n'agit pas et de façon complémentaire, alors je n'agis pas non plus de façon sociale (DEFALVARD, 1999). Ces phénomènes ne sont pas pour autant parfaitement identiques et diffèrent, selon les institutionnalistes américains, en degré d'organisation. Pour MITCHELL (1935), l'institution peut être inorganisée (coutume) ou organisée (famille, entreprise, ou encore l'Etat). Dans une perspective veblenienne, où les institutions régissent à la fois les rapports entre les individus mais aussi entre les individus et le monde matériel, l'organisation apparaît comme une solution institutionnelle à une contrainte technologique (PALLOIX, 2000). Chez Commons, l'institution peut prendre la forme d'organisations actives (going concerns) qui se définissent par un but, des règles de fonctionnement (working rules) et des autorités qui formulent et appliquent ces mêmes règles (CHAVANCE, 2000). Dans ce cadre conceptuel, le terme " organisation" a vocation à rendre compte des institutions qui présentent un caractère organisé ${ }^{6}$.

\section{- 5 - \\ La proximité organisationnelle : une forme particulière de proximité institutionnelle}

Dans son acception traditionnelle, la proximité organisationnelle "lie les agents participant à une activité finalisée dans le cadre d'une structure particulière. (...) [Elle] se déploie à l'intérieur des organisations (firmes, établissements, etc.) et, le cas échéant, entre organisations liées par un rapport de dépendance ou d'interdépendance économique ou financière (entre sociétés membres d'un groupe industriel ou financier, au sein d'un réseau, etc.)" (KIRAT et LUNG, 1995, p. 213). Conservant comme cadre général cette définition, nous cherchons à donner un contenu à cette forme de proximité. Pour ce faire, et suivant la distinction opérée par COMMONS (1934) entre institution et organisation, nous comprenons la proximité organisationnelle comme une forme de proximité institutionnelle. Il s'agit de partager un espace social qui prend cette fois-ci une forme concrète observable par tous (à la différence par exemple d'une coutume).

Les organisations ont vocation à permettre une action collective complexe en coordonnant les actions individuelles. Elles sont le cadre de mises en actes d'idées communes en vue

\footnotetext{
${ }^{6}$ L'utilisation du terme institution dans les expressions «institutions publiques » ou « institutions religieuses », pour ne citer que ces deux exemples, illustre cette compréhension de l'organisation comme une institution particulière.
} 
d'atteindre un objectif, ce qui suppose la résolution de nombreux problèmes de coordination. Ainsi, l'organisation, à l'instar des entreprises, se voit dotée par ses membres de règles de fonctionnement (et des routines afférentes, cf. supra) et d'un "gouvernement" propre qui exerce le pouvoir (BAZZOLI et DUTRAIVE, 2002). De façon générale, l'organisation devient un lieu de production et d'activation de règles et de routines qui permettent de prendre des décisions au nom des acteurs. Ces règles et routines assurent, d'une part une coordination de nature cognitive répondant à la problématique de l'efficacité des actions, et d'autre part une coordination de nature politique répondant à la problématique de la conformité et de la légitimité des mêmes actions réalisées par des acteurs hétérogènes. Dans le premier cas, l'organisation est vue comme une technologie sociale, dans le second, et de façon complémentaire, comme un espace de choix effectués par les acteurs.

\subsection{Coordination cognitive : produire du sens}

De façon générale, les institutions encodent de l'information et suppléer ainsi aux limites de la rationalité (DOUGLAS, 2004). En ce sens, elles permettent la coordination cognitive en réduisant l'incertitude associée à l'action ${ }^{7}$. En effet :

- $\quad$ les institutions établissent des modèles de penser et de faire. En bornant les comportements, elles rendent ces derniers plus semblables et donc plus prévisibles même si des problèmes d'interprétation peuvent se poser. Ainsi la proximité institutionnelle favorise un pronostic adéquat du comportement stabilisé d'autrui ;

- $\quad$ la même analyse peut être faite, non pas pour le comportement lui-même, mais pour les conséquences futures des actions qui sont plus ou moins connues. Ayant été déjà observées, les anticipations des individus sont plus sûres (HODGSON, 2002), l'expérience du passé est intégrée de façon à guider les prévisions (DOUGLAS, 2004);

- dans une perspective veblenienne, le caractère habituel de l'institution présente plusieurs avantages : cette propension à répéter certaines pensées et comportements réduit la recherche d'information et le besoin d'analyse de la situation qui précédent toute action ;

- les institutions partagées et orientées vers une fin sont porteuses de sens, de valeurs, voire d'engagements réduisant l'incertitude. Il s'agit là d'une vision positive de leur finalité, les acteurs les mobilisant non seulement pour satisfaire leurs intérêts individuels ou collectifs, mais aussi parfois parce qu'elles leur semblent justes. Il est alors possible que certains individus continuent de reconnaître des institutions alors qu'ils n'en tirent aucun avantage.

Sur un plan cognitif, les organisations se distinguent des autres institutions en produisant des règles et des routines destinées à la coordination cognitive en vue d'une action collective. Selon BAZZOLI et DUTRAIVE (2002), les règles d'action que chacun élabore face à l'incertitude ont une fonction cognitive indispensable de construction des représentations à travers des expériences empiriques quotidiennes, tout en constituant des guides pour l'action. Il s'agit par les règles de réduire l'incertitude et de sécuriser les anticipations, règles qui ne

\footnotetext{
${ }^{7}$ Selon RIVAUD-DANSET (1998), l'incertitude inhérente à toute action résulte de quatre sources d'erreurs : l'objectivité du monde n'est pas observable; le futur n'est pas correctement inféré ; les conséquences de nos propres actions nous sont partiellement inconnues; nous n'exécutons pas nos actions exactement comme nous nous le représentons.
} 
sont pas incompatibles avec la rationalité. Elles sont même une condition nécessaire à son exercice, en économisant les capacités cognitives pour les activités routinières qui seront dès lors utilisées de façon plus opportunes. "Dans cette perspective, la rationalité n'est pas associée à l'optimisation ni à la délibération consciente au cas par cas; elle correspond à la capacité humaine à concentrer l'activité intelligente sur un nombre limité de facteurs spécifiques pour aboutir à un résultat satisfaisant et repose sur la routinisation des processus cognitifs qui dispensent les individus d'une complète attention et délibération. » (BAZZOLI et DUTRAIVE, 2002, p. 27). La routinisation des actions, loin de s'opposer à l'efficacité, la conditionne.

La notion de routine développée par les approches évolutionnistes de la firme illustre bien la nécessité de cette coordination cognitive. Dans ce cadre théorique, les organisations comme les firmes apparaissent comme des producteurs de routines visant à l'efficacité de l'action. Ces routines sont des "modèles d'interactions qui constituent des solutions efficaces à des problèmes particuliers" (DOSI et al., 1990, p. 243) et qui se composent de séquences codées déterminant un enchaînement de décisions et de comportements (MENARD, 1994) décrivant ce que doivent être les pratiques. Ce sont des habitudes individuelles et collectives qui présentent un caractère très concret et qui stockent un savoir-faire tacite se maintenant dans le temps. Ces routines vont dans le sens d'une réduction de l'incertitude et de sécurisation des anticipations : par leurs caractères mécaniques et automatiques, elles suspendent en effet l'incertitude liée à l'action de l'autre, et apparaissent comme « (...) un mode de résolution pragmatique d'un problème auquel les règles donnent une réponse théorique (...) » (REYNAUD, 2001, p. 64).

\subsection{Coordination politique : produire des choix}

L'institutionnalisme se démarque des compréhensions évolutionnistes des organisations, en ce sens que ces dernières, comme formes institutionnelles, ne doivent pas être réduites à une seule fonction de coordination cognitive. Les institutionnalistes affirment en effet que l'action exprime des choix en relation avec des phénomènes certes de signification et d'évaluation, mais aussi de pouvoir (THERET, 2001). En mettant l'accent sur l'artificialité de l'action et sur l'importance des rapports de pouvoir et des conflits qui en découle, il s'agit de rappeler que le partage peut résulter de rapports de force (TALBOT et KIRAT, 2005). Ainsi, si l'on s'en tient à l'exemple de la firme, la division interne du travail relève à la fois d'une nécessité cognitive de donner un contenu à des tâches, mais aussi d'une nécessité politique d'articuler les actions d'acteurs aux statuts et aux intérêts différents (direction, salariés, syndicats, etc.).

Pour AMABLE et PALOMBARINI (2004), le terme politique renvoie au sens strict à un sous-système social dans lequel sont fixés les choix collectifs qui s'imposent aux acteurs sociaux dans leur ensemble. Au sens large, le politique devient une tentative des acteurs de faire prévaloir par la coordination une organisation sociale conforme à leurs intérêts. Ils essayent par là même d'obtenir et de mettre en œuvre une capacité d'influence (un pouvoir) sur les choix publics, par exemple en définissant les priorités. Dit autrement, la coordination politique implique de prendre des décisions politiques par nature partiales tout en donnant une cohérence à des aspirations contradictoires.

De façon générale, les institutions, en contraignant l'action et en régulant les conflits, permettent la coordination politique de deux façons. D'une part, l'action est contrainte dans sa forme. Se conformer à des institutions revient à adopter des comportements attendus : elles peuvent alors se comprendre comme des prescriptions sociales. Chaque individu se voit imposé un ensemble de statut, dont l'acceptation collective est antérieure à sa propre 
existence, car ils naissent «dans un monde dont le cours est déjà collectif » (DEFALVARD, 2002, p. 28). Concrètement, un agent peut légitimement exercer une domination en créant et en imposant par exemple ses propres règles et objectifs à autrui (pensons au cas de l'autorité dirigeante envers ses salariés). La proximité s'avère porteuse tout autant d'une idée de partage que d'autorité.

D'autre part, les institutions, comme catégories, fonctionnent comme des filtres n'autorisant le traitement que des informations considérées comme acceptables par les acteurs, c'est-à-dire qui n'entrent pas ou faiblement en contradiction avec leurs valeurs. Cette caractéristique réduit temporairement les conflits. Le passage de divers compromis fondant l'action collective devient alors possible. Ces compromis portent notamment sur la compréhension des problèmes existants et sur la façon de les résoudre en vue d'atteindre un but commun. Comme le rappellent AMABLE et PALOMBARINI (2004), le compromis qui résulte d'un processus de coordination politique est obtenu suite à des concessions mutuelles faites par des parties qui ne peuvent faire autrement que de l'accepter. Les raisons du désaccord peuvent persister, le compromis n'étant pas nécessairement considéré comme juste. Que les rapports de force se modifient, et le conflit temporairement apaisé refait son apparition.

Sur un plan politique, BAZZOLI et DUTRAIVE (2002) en analysant la pensée de J. R. Commons caractérisent les organisations, au regard des institutions, de la façon suivante :

- pour exister, une organisation doit avoir une constitution juridique qui renvoie aux buts de celle-ci et exprime les relations de pouvoir et d'autorité. Elle repose sur des règles externes, des règles de droit qui fondent l'existence légale de l'organisation en déléguant un pouvoir normateur et de mobilisation des ressources à ses représentants qui peuvent dés lors contracter au nom de tous. Elle repose aussi sur des règles internes, qui organisent l'exercice du pouvoir, définissent les termes de l'appartenance à l'organisation, répartissent les ressources, permettent la réalisation de choix collectifs et, ajoute HODGSON (2006), établissent des frontières ;

- une organisation, si elle est une structure, est aussi un processus qui permet de produire la volonté, la motivation à participer des membres. Cette motivation est le résultat du désir de chaque individu d'être approuvé par les autres et de l'existence d'une futurité commune aux acteurs, d'une représentation partagée d'objectifs à atteindre grâce aux actions présentes. Partager une proximité organisationnelle est le résultat d'une volonté, d'un acte conscient des acteurs qui peuvent satisfaire leurs intérêts individuels et collectifs par l'atteinte d'une finalité collective ;

- une organisation est une institution si elle perdure dans le temps, malgré le changement de ses membres. Cette longévité, qui autorise la constitution d'une mémoire, est bien entendu fonction de la capacité l'organisation à faire évoluer ses règles pour s'adapter. L'organisation comme institution est bien une entité évolutive.

\subsection{Des coordinations cognitive et politique articulées}

Il ne faut pas voir dans la distinction que nous faisons entre coordination cognitive et coordination politique une catégorisation étanche de phénomènes indépendants. Les niveaux cognitif et politique sont au contraire étroitement articulés. Selon MULLER (2005), au moins deux types de connexions entre les deux champs ainsi définis sont perceptibles. Premièrement, les idées n'existent pas sans les intérêts (les référentiels exprimant la vision du monde des groupes dominants) tandis que les intérêts s'expriment par le prisme des matrices cognitives et normatives (qui analysent le monde et disent comment il doit être). 
Deuxièmement, une communauté de sens permet de coder le réel autorisant une autonomie de fonctionnement : à une même question, il est possible d'apporter des réponses différentes car propres à chaque communauté. La maîtrise du codage du réel est une forme de pouvoir indéniable car "le référentiel constitue bien une contrainte pour les acteurs, à la fois parce qu'il transforme leur perception du monde vécu et disqualifie les anciennes visions $d u$ monde » (MULLER, 2005, p. 172). Il existe des utilisations «stratégiques » des normes et valeurs qui peuvent contribuer au renforcement d'une position, d'autant que le système d'explication du monde (paradigme, référentiel) offre une place privilégiée aux acteurs dont les intérêts s'intègrent au mieux dans le cadre cognitif. Il faut ajouter une troisième articulation possible avec EYMARD-DUVERNAY (2001), qui confère aux représentations et croyances une dimension politique, car elles intègrent à l'action individuelle la volonté de construire un monde commun avec les autres. Cette qualité permet la sélection parmi l'ensemble des représentations des plus légitimes à fonder une société, un collectif.

Ainsi, nous définissons les organisations comme des institutions dotées, d'une part, d'une mémoire composée de règles et de routines comprises comme autant de ressources cognitives mobilisées pour la mise en cohérence des actions individuelles et pour atteindre efficacement les objectifs fixés (coordination cognitive) et, d'autre part, de structures de pouvoir qui ont en charge cette mise en cohérence en incitant à la conformité de ces actions aux règles produites, ce qui permet leur légitimation, et la fixation des choix collectifs (coordination politique). La proximité organisationnelle consistera alors pour des acteurs a priori hétérogènes à intégrer la communauté cognitive, c'est-à-dire à accéder à la mémoire de l'organisation composée de ressources cognitives, et à intégrer la communauté politique, c'est-à-dire à s'insérer dans la structure de pouvoir. Elle est bien une forme particulière de proximité institutionnelle qui consiste, rappelons-le, à partager des idées communes et à assumer des rôles complémentaires.

\section{Conclusion}

Après avoir justifié la conservation des trois dimensions de la proximité, nous avons défini ces différentes formes à travers une grille de lecture associant institutionnalisme américain et philosophie de l'esprit. Nous avons d'abord montré que la proximité géographique est une disponibilité relationnelle. Il est ensuite apparu que la proximité institutionnelle est un effet des institutions. Enfin, nous avons interprété la proximité organisationnelle comme une forme de proximité institutionnelle qui peut se décrire en distinguant, de façon purement analytique, la coordination cognitive de la coordination politique.

Nous venons donc de proposer une analyse statique des proximités. Cette analyse doit s'accompagner dans un second temps d'une lecture dynamique des catégories ainsi définies, en mettant en lumière les jeux de complémentarité et de substituabilité qui s'instaurent entre elles. Ces trois formes de proximité constituent en effet trois piliers de l'action collective, sans qu'il soit pour autant nécessaire de les mobiliser toutes à la fois. En particulier, les proximités organisationnelle et géographique peuvent jouer un rôle important dans un type de coordination, organisée et/ou fondée sur des interactions locales, et occuper une place plus réduite dans un autre, non structurée et/ou bâtie des interactions à distance. Par contre, la proximité institutionnelle apparaît comme une condition nécessaire à toute action collective, étant entendu qu'elle peut varier en intensité. Elle est donc à la fois un effet des institutions et une condition à l'action collective.

Parler de proximité, c'est une façon d'aborder la question des relations qu'ont les individus en société. C'est une approche qui cherche à rendre compte, au moins partiellement, des conditions à l'établissement d'une relation sociale. Elle permet en outre de délimiter l'aire 
d'influence des institutions et par là même de proposer une topologie de l'espace des interactions, définissant un "dedans" et un "dehors". Et de façon indirecte, en qualifiant de plus ou moins proches différents individus et/ou groupes sociaux, la proximité situe ces mêmes individus et ces mêmes groupes les uns par rapport aux autres, dans l'espace concret et dans l'espace des relations. La proximité devient un principe de différenciation sociale. Elle permet de reconnaître que cette différenciation est un caractère socialement construits des agents économiques.

\section{Bibliographie}

AMABLE B., 2005, Les cinq capitalismes. Seuil, Paris.

AMABLE B., PALOMBARINI S., 2004, "Une approche néo-réaliste de l'économie politique", in Douzième rencontre internationales du GERPISA, Comment penser la variété du capitalisme et la diversité des modèles productifs, juin, Paris.

AOKI M., 2006, Fondements d'une analyse institutionnelle comparée. Albin Michel, Paris.

BAZZOLI L., DUTRAIVE V., 2002, "L'entreprise comme organisation et comme institution. Un regard à partir de l'institutionnalisme de J. R. Commons", Economie et Institutions, $\mathrm{n}^{\circ} 1,2^{\mathrm{e}}$ semestre, pp. 4-46.

BAZZOLI L., KIRAT T., 2003, "A propos du réalisme en économie des institutions et ses implications sur l'analyse des fondements juridiques des transactions économiques: Commons versus Williamson", Economie Appliquée, tome LVI, n³, pp. 171-209.

BUSH P., 1987, "The Theory of Institutional Change", Journal of Economic Issues, vol. 21, $\mathrm{n}^{\circ} 3$, pp. 1075-1116.

CAMAGNI R., MAILlAT, D., eds, 2006, Milieux Innovateurs. Théories et politiques. Economica Anthropos, Paris.

CARON A., TORRE A., 2005, "Réflexions sur les dimensions négatives de la proximité", Economie et Institutions, $\mathrm{n}^{\circ} 6$ et $7,1^{\mathrm{er}}$ et $2^{\mathrm{e}}$ semestres, pp. 183-220.

CHAVANCE B., 2000, "Organisations, institutions et système : types et niveaux de règles", in colloque Institutions et Organisations, ERSI-CRIISEA (Université Picardie) et GERME (Université Paris 7), mai, Amiens.

CHAVANCE B., 2007, L'économie institutionnelle. La Découverte, Paris.

COMMONS J. R., 1931, "Institutional Economics", American Economic Review, December, pp. 648-657.

COMMONS J. R., 1934, Institutional Economics. Its Place in Political Economy. MacMillan Company, New York.

COURLET C., SOULAGE B., 1994, Industries, territoires et politiques publiques. L'Harmattan, Paris.

DEFALVARD H., 1999, "Coordination, anticipations et croyances", Economie Appliquée, n³, pp. 7-39.

DEFALVARD H., 2002, "L'Economie des Conventions à l'école des institutions", Economie Appliquée, $\mathrm{n}^{\circ} 4$, pp. 7-33.

DESCOMBES V., 1996, Les institutions du sens. Les éditions de minuit, Paris.

DESCOMBES V., 1998, "L’identification des idées", Revue philosophique de Louvain, 91(1), pp. 86-118.

DESCOMBES V., 2000, "Philosophie des représentations collectives", History of the Human Science, 13(1), pp. 37-49.

DESCOMBES V., 2004, Le complément du sujet. Gallimard, Paris. 
DOSI G., D. TEECE D. et WINTER S., 1990, "Vers une reformulation des frontières de la grande entreprise : la notion de cohérence", Revue d'Economie Industrielle, $\mathrm{n}^{\circ} 51,1^{\mathrm{er}}$ trimestre, pp. 238-254.

DOUGLAS M., 2004, Comment pensent les institutions. La Découverte, Paris. Traduction française de How Institutions Thinks, Syracuse University Press, 1986.

EYMARD-DUVERNAY F., 2001, "Pour un programme d'économie institutionnaliste", Colloque AFSE, septembre.

GILLY J. P., TORRE A., eds, 2000, Dynamique de proximité. L'Harmattan, Paris.

GISLAIN J. J., 2002, "Causalité institutionnelle : la futurité chez J. R. Commons", Economie et Institutions, $\mathrm{n}^{\circ} 1,2^{\mathrm{e}}$ semestre, pp. 47-66.

GROSSETTI M., FILIPPI M., 2004, "Proximité et relations interindividuelles", in Pecqueur B., Zimmermann J. B., eds, Economie de Proximités, Hermès, Lavoisier, Paris.

HAMILTON W., 1993, "Institution", Encyclopedia of the Social Sciences, in HODGSON G., ed, The Economics of Institutions, The International Library of Critical Writings in Economics.

HODGSON G., 2002, "Reclaiming habit for institutional economics", Journal of Economic Psychology, vol. 25, ${ }^{\circ} 5$, pp. 651-660.

HODGSON G., 2003, "John R. Commons and the foundations of institutional economics", Journal of Economic Issues, vol. 37, $\mathrm{n}^{\circ}$ 3, pp. 547-577.

HODGSON G., 2006, "What are institutions ?", Journal of Economic Issues, vol. 40, n 1, pp. $1-25$.

JEANNEAUX P., KIRAT T., 2005, "Proximité, droit et conflits d'usage. Que nous apprend le contentieux judiciaire et administratif sur les dynamiques territoriales ?", Economie et Institutions, $\mathrm{n}^{\circ} 6$ et $7,1^{\text {er }}$ et $2^{\mathrm{e}}$ semestres, pp. 221-248.

KIRAT T., LUNG Y, 1995, "Innovation et proximités : le territoire, lieu de déploiement des processus d'apprentissage", in LAZARIC N., MONNIER J.-M., eds, Coordination économique et apprentissage des firmes, Economica, Paris.

KRUGMAN P., 1991, Geography and Trade. The MIT Press, Cambridge, Mass.

LAGANIER R., VILLALBA B., ZUINDEAU Z., 2002, "Le développement durable face au territoire: éléments pour une recherche pluridisciplinaire", Revue Développement Durable et Territoires, dossier $\mathrm{n}^{\circ} 1$ Approches territoriales du Développement Durable.

LE BRETON D., 2004, L'interactionnisme symbolique. PUF, Paris.

LUNDVALL B.A., 1992, National Systems of Innovation : Towards a Theory of Innovation and Interactive Learning. Pinter Publishers, London.

MAUSS M., 1950, Sociologie et Anthropologie. Presses Universitaires de France, $7^{\mathrm{e}}$ édition premier trimestre 1980, Paris.

MENARD C., 1994, "La nature de l'innovation organisationnelle, éléments de réflexion", Revue d'Economie Industrielle, numéro spécial Économie industrielle : développements récents, juillet, pp. 173 - 192.

MITCHELL W., 1935, "Commons on Institutional Economics", American Economic Review, vol. 25, December, pp. 635-652.

MULLER P., 2005, "Esquisse d'une théorie du changement dans l'action publique", Revue Française de Science Politique, vol. 55, n 1, pp. 155-184.

NORTH D., 1990, Institutions, Institutional Change and Economic Performance. Cambridge University Press, Cambridge.

NORTH D., 1994, "Economic Performance Through Time", American Economic Review, vol. 84, n 3, pp. 359-368.

PALLOIX C., 2000, "Les ouvertures de l'institutionnalisme : apports et limites", in colloque Institutions et Organisations, ERSI-CRIISEA (Université Picardie) et GERME (Université Paris 7), mai, Amiens. 
PECQUEUR B., 1997, "Processus cognitifs et construction des territoires économiques", in Guilhon B., Huard P., Orillard M., Zimmermann J. B., eds, Economie de la connaissance et des organisations, L'Harmattan, Paris.

PECQUEUR B., ZIMMERMANN J. B., 2004, "Introduction. Les fondements d'une économie de proximités" in PECQUEUR B., ZIMMERMANN J. B., eds, Economie de Proximités, Hermès, Lavoisier, Paris.

PORTER M., 1998, "Clusters and the new economics of competition", Harvard Business Review, vol. 76, n6, pp. 77-90.

RALLET A, 1999, "L'économie de proximités", communication à l'école-chercheur INRA, Le Croisic, 8-10 décembre.

RERU, 1993, "Economie de proximités", numéro spécial de la Revue d'Économie Régionale et Urbaine, $\mathrm{n}^{\circ} 3$.

REYNAUD B., 2001, "Suivre des règles dans les organisations", Revue d'Economie Industrielle, $4^{\mathrm{e}}$ trimestre, pp. 53-68.

RIVAUD-DANSET D., 1998, "Le traitement de l'incertitude en situation - Une lecture de Knight", in SALAIS R., CHATEL E., RIVAUD-DANSET D., eds, Institutions et conventions - La réflexivité de l'action économique, Editions de l'Ecole des Hautes Etudes en Sciences Sociales, Paris.

RUTHERFORD M., 1995, "The Old and the New Institutionalism : Can Bridges be Built ?", Journal of Economic Issues, vol. 29, n 2, pp. 443-451.

SEARLE J., 1998, La construction de la réalité sociale. Traduit de l'anglais par C. TIERCELIN, Gallimard, Paris.

SEARLE J., 2005, "What is an institution? ", Journal of Institutional Economics, vol. 1, n¹, pp. $1-22$.

SJÖSTRAND S.-E., 1995, "Towards a theory of institutional change", in GROENEWEGEN J., PITELIS C., SJÖSTRAND S.-E., eds, On Economics Institutions : Theory and Applications, European Association for Evolutionary Political Economy, Cambridge.

TALBOT D., KIRAT T., eds, 2005, "Proximités et Institutions : nouveaux éclairages", Economie et Institutions, $\mathrm{n}^{\circ} 6$ et 7, $251 \mathrm{p}$.

THERET B., 2001, "Saisir les faits économiques : la méthode Commons", in Lecture de John $R$. Commons, Cahiers d'économie politique, $\mathrm{n}^{\circ} 40-41$, Paris, L'Harmattan.

TORRE A., FILIPPI M., eds, 2005, Proximité et changements socio-économiques dans les mondes ruraux. INRA Editions, Paris.

TORRE A., RALLET A., 2005, "Proximity and localization", Regional Studies, vol. 39, n¹, pp. 47-60.

VEBLEN T., 1898, "Why is economics not an evolutionary science ?", Quaterly Journal of Economics, July, pp. 373-397.

VEBLEN T., 1899, "Preconceptions of Economic Science", II, Quaterly Journal of Economics, July, pp. 396-426.

WILLIAMSON O. E., 1994, Les institutions de l'Economie. Inter Editions, Paris. Edition française de The Economic Institutions of Capitalism, The Free Press, Macmillan, New York, 1985.

WILLIAMSON O. E., 1996, "Revisiting Legal Realism: The Law, Economics, and Organization Perspective", Industrial and Corporate Change, vol. 5, n², pp. 383-420. 